\title{
Outcomes of Minimally Invasive, Small-Incision Unilateral Recession-Resection Surgery and Establishment of an Artificial Intelligence Predictive Mode
}

\section{Xin Xu}

Peking University People's Hospital

Lili Guo

Peking University People's Hospital

\section{Zequn Miao}

Peking University People's Hospital

\section{Qianru Ouyang}

Peking University People's Hospital

Jingyi Zhang

Yantai Yuhuangding Hospital

\section{Yan Li}

Peking University People's Hospital

\section{Qingyu Meng}

Peking University People's Hospital

\section{Shuting Liang}

Peking University People's Hospital

\section{Yu Cao}

Peking University People's Hospital

\section{Renhao Tang}

Bejing University of Technology

\section{Wensi Wang}

Bejing University of Technology

Lejin Wang (D 13911822021@163.com )

Peking University People's Hospital

\section{Research Article}

Keywords: intermittent exotropia, strabismus surgery, artificial intelligence, predictive mode

Posted Date: April 20th, 2021 
DOI: https://doi.org/10.21203/rs.3.rs-415173/v1

License: (c) (1) This work is licensed under a Creative Commons Attribution 4.0 International License. Read Full License 


\section{Abstract}

BACKGROUND: To observe the outcomes after minimally invasive, small-incision unilateral recessionresection (R\&R) surgery and to establish an artificial intelligence (AI)-based operation design predictive model for modified strabismus surgery.

METHODS: Basic intermittent exotropia (IXT) patients with an exodeviation angle between 20 and 85 prism diopters underwent the modified R\&R surgery. The core procedure of the operation is to separate and completely resect the check ligaments. After at least six months of follow-up, the exodeviation angle, postoperative success rate, pre- and postoperative fusional function and change in stereopsis were observed retrospectively. The predictive model was established based on the successful cases; 38 related factors were entered into the model. The machine learning algorithm LightGBM was used to train the model.

RESULTS: In all, 113 patients were included: 48 males and 65 females. The average follow-up time was 0.749 years. The surgical success, overcorrection and undercorrection rates were $85.8 \%(97 / 113), 2.7 \%$ $(3 / 113)$ and $11.5 \%(13 / 113)$, respectively. There was a significant improvement in the fusional range from $14.61 \pm 8.43^{\circ}$ preoperatively to $22.70 \pm 5.72^{\circ}$ postoperatively $(t=-9.726, P<0.001)$. Near stereoacuity improved more than $60 \%$ postoperatively. For distance stereoacuity, $47(41.59 \%)$ patients showed recovery, with no significant difference regarding the presence or absence of stereoacuity (chi-square test, $\left.c^{2}=3.762, P>0.05\right)$. The Al-based operation design predictive model was preliminarily established; the accuracy reached $95 \%$.

CONCLUSION: Minimally invasive, small-incision unilateral R\&R surgery is effective for treating basic IXT, with a high postoperative success rate and good binocular functional recovery. This could be a better surgical design for treating basic IXT. A preliminary Al predictive model based on this operation was established.

\section{Background}

Intermittent exotropia (IXT) is a common disease in pediatric ophthalmology, with a cumulative incidence of $1.0 \%$ in the first decade of life ${ }^{1}$. It is characterized by the periodic deviation of one eye, typically including closing one eye in bright sunlight and frequently occurring when the child is sleepy or inattentive. The majority of patients with IXT have sensory dysfunction, including the loss of fusion function and stereopsis ${ }^{2,3}$. Basic IXT is the most frequently diagnosed form of IXT. This type is mainly treated by recession-resection (R\&R) surgery, aiming to correct the eye position and maintain binocular function. According to the literature, the success rate varies from $40-83 \%{ }^{4-10}$. However, traditional surgical procedures do not fully expose the extraocular muscles; large incisions and large surgical doses tend to cause slow healing postoperatively, poor recovery of appearance, and complications such as adhesions and restricted eye movement. Through intraoperative observation, the extraocular muscle check ligaments may be the key factor for postoperative adhesion formation, eye movement restriction 
and postoperative exotropic drift ${ }^{11,12}$. Therefore, in this study, the traditional unilateral R\&R operation was improved by fully resecting the pulleys of the extraocular rectus during the operation, and the clinical effect of this approach was evaluated. On this basis, using artificial intelligence (Al), the surgical design used in this research was digitized through mathematical algorithms, and a predictive model was established, paving the way for the application and popularization of the improved surgery.

\section{Methods}

A retrospective study was conducted in 113 patients who visited Peking University People's Hospital with IXT from March 2018 to January 2020. Informed consent was obtained from the patients or their guardians, and the research protocol was approved by the Medical Ethics Committee of Peking University People's Hospital (permit number: 2019PHE031).

Patients with basic IXT (the near deviation was within 10 prism diopters (PD) of the distance deviation after unilateral eye occlusion for more than 30 minutes) less than 18 years old were included. The angle of deviation of the included patients was in the range of 20 to 85 PD. In these IXT patients, preoperative deviation manifested frequently and required surgical treatment. All subjects had normal vision or corrected-to-normal vision. Patients were able to undergo ophthalmologic examinations and complete stereopsis tests. To obtain accurate measurements in the prism and alternative cover tests and the binocular vision test, the lower limit of age for inclusion was set at three years.

The exclusion criteria were as follows: over 18 years old; amblyopia; simultaneous vertical strabismus or oblique muscle abnormality; lateral incomitance; organic eye disease or nystagmus; and history of previous strabismus, intraocular surgery or botulinum toxin injection. Patients with neurologic disease were also excluded.

Examination and data collection: All patients underwent a complete ophthalmologic examination before the operation, including examination of the best-corrected visual acuity and intraocular pressure, slit-lamp anterior-segment examination and indirect ophthalmoscopy. The data collected included sex, refractive error, age at surgery, duration of exotropia, dominant eye, and deviation when fixating on a near target (33 $\mathrm{cm}$ ) and a distant target $(6 \mathrm{~m})$ pre- and postoperatively. The refractive error was detected by an ARK-510A automated refractor (NIDEK Co., Ltd., Japan) after mydriasis. Tropicamide phenylephrine eye drops (Santen Pharmaceutical Co., Ltd., Japan) were applied to induce mydriasis. For children with poor coordination, retinoscopy was performed with a YZ24 retinoscope (Suzhou Liuliu Vision Technology Co., Ltd.). The maximum angle of deviation was measured by applying the alternate cover and prism tests with loose prisms. Binocular function was investigated, and the results were recorded. The preoperative and postoperative near stereoacuity were determined in all patients by the Titmus (Titmus Optical Co., Petersburg, Virginia, USA) stereoacuity test. A stereoacuity $\leq 60 \mathrm{~s}$ of arc was defined as bifixation, and a stereoacuity $\leq 800 \mathrm{~s}$ of arc was defined as gross stereopsis. The results of patients who exhibited no stereopsis were recorded as nil for the purpose of calculation. The pre- and postoperative fusional function were tested in all patients by a synoptophore (Inami \& Co., Japan). The simultaneous point, 
fusion function, and distant stereoscopic vision were measured and recorded using I, II, and III synoptophore drawing slides. If there was no simultaneous point, the rest of the results were recorded as nil. When the fusion function was measured, convergence fusion and divergence fusion were measured three times, and the average values were recorded. For the distant stereoscopic inspection, qualitative measurement was performed. The duration of follow-up was at least half a year. The outcome was considered successful if the postoperative angle of deviation was between 10 PD of exodeviation and 5 PD of esodeviation.

Surgical procedures: Minimally invasive, small-incision R\&R surgery was routinely chosen for the nondominant eye. All operations were performed by the same surgeon.

Recession of lateral rectus muscle: A 4- to 5-mm-long Parks fornix incision was made under an OMS-710 ophthalmic operating microscope (Topcon Co., Ltd., Japan) at the inferior temporal bulbar conjunctiva. The muscle sheath, intermuscular membrane and check ligaments were fully separated to approximately 2-3 $\mathrm{mm}$ after the expected recession, and the check ligaments attached to the sheath were completely resected. A 6 - 0 Vicryl double-needle absorbable suture (produced by Ethicon Company, USA) was used to make suture loops $1.5 \mathrm{~mm}$ from the muscle termination. The lateral rectus muscle was cut $1 \mathrm{~mm}$ before the suture and sutured to the superficial sclera where the amount of recession was expected (Figs. 1 and 2).

Resection of medial rectus muscle: A Parks cul-de-sac approach was performed under an ophthalmic operating microscope at the inferior nasal bulbar conjunctiva. The same method was used to separate the pulleys around the medial rectus muscle. The estimated amount of the medial rectus was amputated, and the muscle was sutured to the location of the rectus insertion.

Surgical design: The surgical dosage was determined by the maximum angle of deviation within near and distance strabismus. When the angle of strabismus was - 20 PD -35 PD, the recession of the external rectus and the resection of the medial rectus were designed to be $5 \mathrm{~mm}$ and $4 \mathrm{~mm}$ ("recess 5 , resect 4") or $6 \mathrm{~mm}$ and $3 \mathrm{~mm}$ ("recess 6 , resect 3"), respectively; when the angle of deviation was - 36 PD -50 PD, the designed dosage was "recess 6, resect 4"; when the exotropic degree was - 51 PD -75 PD, the designed dosage was "recess 7, resect 4", and when the degree of strabismus was - 76 PD -90 PD, the designed dosage was "recess 7 , resect 5 ". The above are just the basic guidelines for the surgical design, which is not set in stone. According to the patient's eye position control, binocular visual function damage and other influencing factors, the surgeon can make certain adjustments to the surgical design.

Prediction method based on Al: In this study, Python was used to build this model, and the LightGBM model (a machine learning algorithm based on the decision tree model) was used to predict the dosage of surgery. First, case collection was carried out, and the data of 97 patients with satisfactory results were collected. Finally, all preoperative data were recorded from the hospital's medical record database, including sex, age, time of onset, history of systemic disease, previous eye disease, refractive error, visual acuity with and without correction, dominant eye, binocular visual function, negative relative accommodation, positive relative accommodation, anesthesia, and surgical volume, among others. There 
was a total of 38 indicators. Due to the different data units, the data were standardized to be nondimensional. The numerical data were stored directly, with precision to two decimal places; the textual data were classified according to the keywords and quantified into enumeration data; the null values were maintained with default values. Data standardization was carried out after the removal of dimensions. The standardization formula was

$$
Y=\frac{X-\bar{X}}{S}
$$

(Y: standardized data; $\mathrm{X}$ : variable; $\mathrm{X}$ : mean value; $\mathrm{S}$ : standard deviation). The hyperparameter settings in the LightGBM model were as follows: the boosting type was 'dart'; the learning rate was 0.1 ; the depth of the tree was determined by autoadaptation; and the number of classes, estimators, and leaves was 8192, 100 and 8 , respectively. Then, $90 \%$ of the data from the cases were randomly selected as the training set, and the remaining $10 \%$ were selected as the test set. Finally, the training set data were input into the LightGBM ${ }^{13}$ model for parameter training, and the trained model was saved. After obtaining the model, the doctor could input new patient data into the model to obtain the dosage of surgery. A flow chart of the establishment of the predictive model is shown below. (Fig. 3)

SPSS version 22.0 (SPSS, Chicago, IL, USA) was used for data processing. The age at onset, course of disease, age at surgery, refractive error, and angle of strabismus (PD) are expressed as the mean \pm standard deviation; the success rate and near stereoscopic improvement rate are expressed as percentages. The preoperative and postoperative fusion function and near stereoacuity were compared by paired T-test. The chi-square test was performed to compared the presence and absence of distance stereoacuity. Differences were considered statistically significant when $\mathrm{P}<0.05$.

\section{Results}

During the study period, 113 patients with basic IXT were enrolled, and they all underwent minimally invasive, small-incision unilateral R\&R surgery. Of these patients, 48 (42.5\%) were male, and $65(57.5 \%)$ were female. The mean age at surgery was $8.19 \pm 3.2$ years among males and $8.18 \pm 3.01$ years among females. The overall age at onset was $5.5 \pm 2.7$ years (range, $0.5-11.5$ years), the course of disease ranged from 0.5 to 9.5 years, and the average disease duration was 2.7 years. The mean preoperative strabismus was $-32.57 \pm 13.40 \mathrm{PD}$, with a range of -20 to $-85 \mathrm{PD}$. All patients were followed up for more than half a year; the average follow-up time was 0.749 years, with a maximum follow-up of 3.5 years. At the last follow-up, there was an overall success rate of $85.5 \%$ (97/113). Residual exotropia was present in $11.5 \%(13 / 113)$ of patients, and $2.7 \%(3 / 113)$ of patients had consecutive esotropia. The descriptive characteristics of the patients in our study are shown in Table 1. 
Table 1

Characteristics of gender groups in this study.

\begin{tabular}{|c|c|c|c|}
\hline Variables & Male $(n=45)$ & Female $(n=68)$ & Entire $(n=113)$ \\
\hline Age of onset(Y) & $5.52 \pm 2.94$ & $5.48 \pm 2.58$ & $5.5 \pm 2.7$ \\
\hline Course of disease $(\mathrm{Y})$ & $2.66 \pm 1.89$ & $2.73 \pm 2.12$ & $2.7 \pm 2.0$ \\
\hline Age at surgery $(\mathrm{Y})$ & $8.19 \pm 3.29$ & $8.18 \pm 3.01$ & $8.18 \pm 3.12$ \\
\hline \multicolumn{4}{|c|}{ Preoperative spherical equivalent(D) } \\
\hline Operative eye & $-0.36 \pm 2.10$ & $-0.38 \pm 2.13$ & $-0.37 \pm 2.12$ \\
\hline Nonoperative eye & $-0.26 \pm 1.52$ & $-0.38 \pm 2.15$ & $-0.32 \pm 1.90$ \\
\hline Titmus before surgery(arcsec) & $102.50 \pm 162.66$ & $90.92 \pm 146.31$ & $95.84 \pm 153.18$ \\
\hline \multicolumn{4}{|c|}{ Preoperative angle of strabismus (PD) } \\
\hline At near & $-32.44 \pm 12.88$ & $-33.41 \pm 15.92$ & $-33.00 \pm 14.65$ \\
\hline At distance & $-30.06 \pm 12.86$ & $-31.49 \pm 16.20$ & $-30.88 \pm 14.66$ \\
\hline \multicolumn{4}{|l|}{ Preoperative fusion function $\left({ }^{\circ}\right)$} \\
\hline Fusion range & $12.50 \pm 10.21$ & $13.25 \pm 8.42$ & $14.61 \pm 8.43$ \\
\hline Convergence fusion & $8.75 \pm 7.66$ & $8.92 \pm 6.24$ & $8.85 \pm 6.86$ \\
\hline Divergence fusion & $3.73 \pm 2.86$ & $4.23 \pm 3.22$ & $4.02 \pm 3.08$ \\
\hline
\end{tabular}

- Surgical formula and postoperative outcomes

As shown in Table 2, fifty-five participants underwent lateral rectus recession ( $5 \mathrm{~mm}$ ) and medial rectus resection ( $4 \mathrm{~mm}$ ) surgery; satisfactory outcomes were achieved in 46 patients, overcorrection occurred in 1 patient, and undercorrection occurred in 8 patients. The mean preoperative strabismus was $-27.90 \pm$ 6.71 PD (mean \pm SD). Twenty-seven patients underwent lateral rectus recession $(6 \mathrm{~mm})$ and medial rectus resection ( $3 \mathrm{~mm}$ ) surgery; undercorrection was observed in 1 of these patients at the last follow-up, and the other patients showed satisfactory outcomes. The mean angle was $-26.67 \pm 6.93$ PD. In addition, 20 patients underwent lateral rectus recession $(6 \mathrm{~mm})$ and medial rectus resection $(4 \mathrm{~mm})$ surgery; 2 of whom showed unsatisfactory outcomes, undercorrection in 1 and overcorrection in 1 . The remaining 11 patients with greater exotropic degrees (over - 50 PD) underwent surgery with a larger dosage design; 5 underwent lateral rectus recession $(7 \mathrm{~mm})$ and medial rectus resection $(4 \mathrm{~mm})$, and 6 underwent lateral rectus recession $(7 \mathrm{~mm})$ and medial rectus resection $(5 \mathrm{~mm})$. 
Table 2

Surgical dosage and the last follow-up postoperative outcome

\begin{tabular}{|lllll|}
\hline $\begin{array}{l}\text { Recession Lateral Rectus/Resect } \\
\text { Medial Rectus, mm }\end{array}$ & $\begin{array}{l}\text { Number } \\
\text { (Male/Female) }\end{array}$ & Outcome & & Success(n) \\
\cline { 5 - 5 } & & $\begin{array}{l}\text { Consecutive } \\
\text { esotropia(n) }\end{array}$ & & $\begin{array}{l}\text { Residual } \\
\text { exotropia(n) }\end{array}$ \\
\hline $5.0 / 4.0$ & $55(20 / 35)$ & 1 & 25 & 8 \\
\hline $6.0 / 3.0$ & $27(14 / 13)$ & 1 & 17 & 1 \\
\hline $6.0 / 4.0$ & $20(12 / 8)$ & 1 & 3 & 2 \\
\hline $7.0 / 4.0$ & $5(1 / 4)$ & 0 & 6 & 0 \\
\hline $7.0 / 5.0$ & $6(1 / 5)$ & 0 & 97 & 13 \\
\hline Total & $113(48 / 65)$ & 3 & & 2 \\
\hline
\end{tabular}

- Improvement of fusion function

In this study, 13 children were unable to understand and cooperate with the synoptophore examination due to their young age. Therefore, the synoptophore fusion function of 100 patients was analyzed. The absolute values of the mean fusion range of 100 patients before and after the operation were $14.61 \pm$ $8.43^{\circ}$ and $22.70 \pm 5.72^{\circ}$, respectively; the difference between the two was statistically significant $(\mathrm{t}=-9.726, \mathrm{P}<0.001)$. Both convergence fusion and divergence fusion were significantly improved after the operation $(t=-9.473, P<0.001 ; t=-5.005, P<0.001)$. Detailed information is shown in Table 3.

Table 3

Comparison of fusion function before and after surgery

\begin{tabular}{|llll|}
\hline Pre-Po & mean \pm SD & $\mathbf{t}$ & p value \\
\hline Range of fusion & $-8.09 \pm 0.83$ & -9.726 & $<0.001^{\star}$ \\
\hline Convergence fusion & $-6.63 \pm 0.69$ & -9.473 & $<0.001^{\star}$ \\
\hline Divergence fusion & $-1.52 \pm 0.30$ & -5.005 & $<0.001^{\star}$ \\
\hline Abbreviations: Pre, pre-operative; Po, post-operative. \\
\hline
\end{tabular}

- Comparison of near and distant stereoacuity

The postoperative near stereoacuity was mainly distributed from $40 \mathrm{~s}$ of arc to $100 \mathrm{~s}$ of arc, and the majority of patients improved after therapy (preoperative vs. postoperative; $101.31 \pm 143.20$ vs. $51.92 \pm$ 42.18). Seventy-seven (68.1\%) patients showed $40 \mathrm{~s}$ of arc at the last follow-up. Ten patients who did not have preoperative near stereoacuity showed improved function within the range of $40 \mathrm{~s}$ arc to $200 \mathrm{~s}$ arc. A significant improvement in near stereoacuity was observed in $60.18 \%(n=68)$ of the patients at the last follow-up (Fig. 4). 
For distance stereoacuity, 47 (41.59\%) patients showed recovery after surgery. The chi-square test was performed to examine the presence or absence of distal stereoscopic vision before and after the operation, and the difference was not statistically significant $(X 2=3.762, P>0.05)$.

- Accurate prediction by the Al-based operation volume model

In this part of our study, the surgical data of 97 successful cases were analyzed by statistical methods, and 38 dimensions of characteristic data were extracted. Then, the data of these 38 dimensions are used as input to train the LightGBM model. The experimental results showed that with an increase in the amount of data in the training set, the prediction accuracy improved. When $90 \%$ of the data were used in the training set and the remaining 10 percent were used as the prediction set, the predictive accuracy reached $95 \%$ (Fig. 5).

\section{Discussion}

Currently, there are two main types of surgical methods for IXT: bilateral lateral rectus recession (BLR) and unilateral lateral rectus recession and medial rectus resection (R\&R). Because the principle of monocular surgery is to reduce abduction and enhance convergence function, it is more suitable for basic IXT than symmetric lateral rectus recession, which is more effective in correcting divergence excess exotropia. This recommendation was confirmed in a randomized control trial performed by Kushner ${ }^{7}$. The success rate of unilateral surgery was $82 \%$, which was significantly higher than that of bilateral surgery (52\%) among patients with basic IXT. In 1972, microscope-assisted technology was first used in strabismus surgery ${ }^{14}$. Compared with traditional surgery performed by the naked eye, this method can clearly show the subtle structures and anatomical layers, allowing more complete separation and preservation of ciliary blood vessels and reducing the incidence of serious complications, including scleral penetration, muscle slippage, hematoma, and incision malpositioning ${ }^{15,16}$.

There are still some shortcomings in the current surgical design. Most operations fail to fully separate the check ligament around the extraocular muscle, and the designed surgical dosage is large, which can easily lead to adhesion formation, extraocular movement limitation and a high rate of exotropic drift after the operation ${ }^{17}$. Burain and Spivey ${ }^{18}$ believed that different subtypes of IXT should be treated with different procedures and that R\&R surgery should be the first choice for the basic type. Therefore, according to this principle, based on the traditional R\&R operation, the minimally invasive microscopeassisted technique combined with the Parks cul-de-sac approach was used to improve the operation. In all patients, the extraocular musculature pulley and other connective tissue were fully resected, and the extraocular muscles were thoroughly separated and exposed. These procedures not only reduced the surgical dosage and alleviated muscle damage but also decreased the incidence of complications. 
The reported results of R\&R surgery vary. In 2006, Jin ${ }^{19}$ observed 66 patients with basic IXT who underwent traditional R\&R surgery. The preoperative deviation ranged from 12 to $45 \mathrm{PD}$, and the operative age was over 8 years old. After an average follow-up period of more than 15 months, 55 of the 66 patients (83.3\%) showed a satisfying outcome. The American Pediatric Eye Disease Investigator Group 20 performed a multicenter clinical trial of 96 IXT patients who underwent R\&R surgery. The proportion of participants showing suboptimal surgical outcomes by 3 years was $37 \%$. A suboptimal outcome was defined as follows: $\geq 10$ PD of exotropia or $\geq 6$ PD of esotropia at distance or near using the simultaneous prism and cover test (SPCT); loss of 2 octaves or more of stereoacuity from baseline, at any masked examination; or reoperation. However, because the method of outcome evaluation was based on the SPCT, the success rate may have been overestimated. Choi ${ }^{21}$ retrospectively reviewed the medical records of 125 children who had undergone unilateral R\&R surgery for large-angle ( $\geq 40 \mathrm{PD})$ or moderate-angle ( $\geq 20$ and $<30$ PD) IXT with a postoperative follow-up period of at least 6 months. After the 1 -year follow-up, the success rate was $55 \%$ in the large-angle group and $75 \%$ in the moderate-angle group. Surgical success was defined as an alignment between 10 PD of exodeviation and 5 PD of esodeviation at both distance and near. At 6 months after the operation, the overall success rate in this study (85.5\%) was higher than that in the abovementioned studies. In view of the differences in the study population, surgical dosage and follow-up time, it is meaningless to compare the advantages and disadvantages of traditional surgery and this procedure among different study samples. However, if the evaluation index focuses only on the surgical success rate, the curative effect of the operation used in this study is ideal for basic IXT, with a range of exotropia from 20 to 85 PD.

The reduction in the fusion range caused by convergence fusion dysplasia and divergence fusion overdevelopment is the main reason for the formation of IXT. The convergence and divergence fusion functions can be evaluated by synoptophore examination. IXT patients are reported to have high convergence fusional amplitudes ${ }^{22}$ because they use convergence fusion to compensate for the deviation. The fusional convergence function was studied using a 1- to 40-PD fixed, horizontal prism bar, different from our study. The mean convergence reserves were significantly lower at distance fixation for children with IXT than for children with normal vision ( $7 \pm 8$ PD vs $17 \pm 7 \mathrm{PD}, \mathrm{P}<0.001$ ), and the mean fusion recovery point was significantly lower at distance for children with IXT than for children with normal vision ( $2 \pm 5 \mathrm{PD}$ vs $11 \pm 6 \mathrm{PD}, \mathrm{P}<0.0001)$. $\mathrm{Mi}^{23}$ found that the degree of convergence fusion increased from $22.36 \pm 3.45^{\circ}$ to $26.00 \pm 2.77^{\circ}$ at 6 months after conventional R\&R surgery. This is consistent with the improvement in the fusion function of the patients in our study. Therefore, our results indicate that the improvement in the postoperative fusion range and convergence fusion function is the main reason for the satisfactory outcomes of this operation.

Stereoacuity is the highest rank of binocular vision based on simultaneous fusion function, which determines the ability to identify the three-dimensional position of surrounding objects. This ability is not available at birth, and only after the visual environment stimulates the fovea can it gradually develop to maturity ${ }^{24}$. In daily life, basic IXT patients alternately exhibit normal ocular alignment and exotropia. Therefore, the patient's stereo vision also appears alternately. In IXT patients, normal near stereoacuity 
shows that they have good control, while decreased or even absent near stereopsis function indicates declining control; thus, near stereoacuity is considered as an objective index to evaluate the control of IXT patients ${ }^{25}$. A large number of studies have shown that the surgical correction of eye position can effectively improve near stereoacuity ${ }^{26,27}$. Wu ${ }^{28}$ found through a prospective study that almost all patients with basic IXT could recover near stereoscopic vision after surgery and that $74 \%$ of IXT patients could reach bifixation (stereoacuity $<60 \mathrm{~s}$ of arc). During the 6 months of follow-up in our study, the near stereoscopic function of the patients increased by more than $60 \%$, indicating that this operation can not only retain the remaining stereo vision but also improve and even restore the damaged stereoacuity.

Traditional surgical dosage design and its shortcomings: Traditional R\&R surgery uses the Parks formula to design the surgical dosage ${ }^{29}$; that is, "recession 5 , resection 4 " in the nondominant eye can correct 20 PD of strabismus. After that, an additional $1 \mathrm{~mm}$ of R\&R is required to correct 8 PD - 10 PD. The formula is simple and easy to understand, but when the strabismus degree is more than $60 \mathrm{PD}$, extralong rectus recession $(>7 \mathrm{~mm})$ or rectus resection $(>5 \mathrm{~mm})$ is needed. This will not only reduce the corrective effect but also increase the occurrence of severe complications, such as limited ocular motility.

Improvement of the surgical volume: Clinically, there is no simple linear correlation between the surgical volume and strabismus (point-to-point); in addition, the control of near and distant ocular alignment varies among strabismus patients, so the range estimation of the surgical dosage (point-to-line) may be more suitable for surgical design in the treatment of basic IXT. Weakening abduction and strengthening convergence are the principles of R\&R surgery. Recession of the lateral rectus muscle only displaces the tendon and does not destroy the biomechanical properties of the muscle. The weakened strength is easier to estimate, and the prognosis is more controllable. Therefore, when the lateral rectus is recessed from $5 \mathrm{~mm}$ to $7 \mathrm{~mm}$, resection of the medial rectus should be reduced accordingly. The modified operation completely separated the extraocular ligament and other connective tissue on the basis of micromanipulation, which weakened its effect on the biomechanics of the extraocular muscle. Through analysis of the relationship between the surgical volume and exotropia, we found that the "recess 5 , resect 4" surgical dosage of the traditional R\&R operation could correct - 20 PD of exotropia, while the maximum angle that could be corrected in this study was - 35 PD. The angle of strabismus that could be corrected by a surgical dosage of "recess 7 , resect 5 " was approximately - 80 PD in our research. Therefore, the design scheme not only greatly reduces extent of the surgery but also expands the scope of surgical indications. Additionally, the surgical trauma of patients and occurrence of postoperative adhesion, eye movement limitation and other complications should be reduced as much as possible, and the success rate of the operation should be improved.

The learning curve of IXT surgery is steep, and doctors often need years of clinical experience to become competent. This is due to differences in surgical procedures and surgical experience. Our surgical method completely separates and exposed the check ligament around the extraocular muscle. Second, the judgment of the patient's condition varies, and the patient's age, refractive error and control of far and near exotropia and other factors will affect the operation. In addition, the anatomical structure and biomechanical strength of the extraocular muscle found in the course of the operation may also influence 
the outcome of the surgery. In traditional surgery, the operative method is selected according to the exotropia type ${ }^{7,30}$, and then the surgical dosage is calculated according to the degree of deviation. Do factors other than far or near exotropia have an impact on the design of the surgical volume? The answer is certain, but there are no unified or comprehensive standards for quantification or calculation. For the time being, the establishment of an Al-based predictive model can answer the above question. We put all the factors that may affect a patient's operation into a "black box" and let the computer perform calculations, find the logic, digitize the results, and finally output a predicted surgical dosage. In this study, a total of 38 factors were entered into this model, and the predictive accuracy reached $95 \%$. Traditionally, it is believed that the surgical design is determined only by the angle of strabismus. We analyzed the contribution rate of these factors to the construction of the model. With the increase in influencing factors, the contribution rate gradually approached 1 . This indicates that the 38 indicators jointly affect the final output. This method converts the preoperative indexes into a digital operation model, which is convenient for inexperienced or nonpediatric ophthalmologists to learn and use, reduces the difficulty of learning, and facilitates surgical design and execution. Al-assisted clinical diagnosis and treatment are gradually becoming popular, and their application in ophthalmology is mainly embodied by image processing and feature extraction in imaging diagnosis ${ }^{31,32}$. Such a predictive model is urgently needed.

There are still many shortcomings in this study. This was a retrospective study, and in most cases, the postoperative follow-up duration was only half a year, preventing long-term observation of the postoperative effect. The detection of fusion function using a synoptophore has seldom been applied in clinical research in the past. In the future, prospective, clinical studies with a longer follow-up period are needed, and binocular vision tests should be more comprehensive. The predictive model is based on previous surgical data; the amount of data in the database is still relatively small, and the predictive stability is insufficient, so it is necessary to strengthen the practical application of this model in clinical practice in the future.

In summary, after minimally invasive, small-incision unilateral R\&R surgery for basic IXT, the success rate was high, and the postoperative fusion function and stereoscopic function were significantly improved, demonstrating the efficacy of this procedure for treating basic IXT of -20 -85 PD. The Al-based strabismus surgery design model digitizes the influencing factors of the surgical design, reduces the difficulty of learning, and can improve work efficiency. In the future, the accuracy achieved by Al-based diagnosis and treatment will not be lower than that achieved by doctors, and attention should be paid to data standards, collection, analysis and sharing. A large number of standard and diverse data sets can increase the reliability and efficiency of Al training results. The development of Al will greatly reduce the pressure on doctors and children and promote the development of medical research in new directions

\section{Abbreviations}

IXT: Intermittent exotropia 
R\&R: Recession-resection
Al: Artificial intelligence
PD: Prism diopter

\section{Declarations}

Ethics approval and consent to participate: Written informed consent was obtained from all the patients' parents/legal guardians, all methods were carried out in accordance with the Declaration of Helsinki, and the research protocol was approved by the Medical Ethics Committee of Peking University People's Hospital (permit number: 2019PHE031).

Consent for publication: Not Applicable

Availability of data and materials: The data used to support the conclusion of this study are available from the corresponding author upon request

Competing interests: The authors indicate no financial support or conflicts of interest

Funding: This work was supported, in part, by the National Science Foundation of China Grant $(31427801,81470665)$

Authors' Contributions: Author contribution: Lejin Wang, Wensi Wang, Lili Guo contributed to the conception of the study; Qainru Ouyang , Jingyi Zhang, Shuting Liang are responsible for data collection; Xin Xu, Zequn Miao, Qingyu Meng contributed significantly to analysis and manuscript preparation; Renhao Tang, Yu Cao, Yan Li helped perform the analysis with constructive discussions.

Acknowledgements: Not applicable

\section{References}

1. Govindan M, Mohney BG, DiehI NN, Burke JP. Incidence and types of childhood exotropia: a population-based study. Ophthalmology. 2005;112(1):104-8.

2. Stathacopoulos RA, Rosenbaum AL, Zanoni D, Stager DR, McCall LC, Ziffer AJ, et al. Distance stereoacuity. Assessing control in intermittent exotropia. Ophthalmology. 1993;100(4):495-500.

3. O'Neal TD, Rosenbaum AL, Stathacopoulos RA. Distance stereo acuity improvement in intermittent exotropic patients following strabismus surgery. Journal of pediatric ophthalmology and strabismus. 1995;32(6):353-7; discussion 8.

4. Buck D, Powell CJ, Sloper JJ, Taylor R, Tiffin P, Clarke MP. Surgical intervention in childhood intermittent exotropia: current practice and clinical outcomes from an observational cohort study. The British journal of ophthalmology. 2012;96(10):1291-5. 
5. Kim HJ, Choi DG. Clinical analysis of childhood intermittent exotropia with surgical success at postoperative 2 years. Acta ophthalmologica. 2016;94(2):e85-9.

6. Richard JM, Parks MM. Intermittent exotropia. Surgical results in different age groups. Ophthalmology. 1983;90(10):1172-7.

7. Kushner BJ. Selective surgery for intermittent exotropia based on distance/near differences. Archives of ophthalmology (Chicago, III: 1960). 1998;116(3):324-8.

8. Ing MR, Nishimura J, Okino L. Outcome study of bilateral lateral rectus recession for intermittent exotropia in children. Ophthalmic surgery and lasers. 1999;30(2):110-7.

9. Maruo T, Kubota N, Sakaue T, Usui C. Intermittent exotropia surgery in children: long term outcome regarding changes in binocular alignment. A study of 666 cases. Binocular vision \& strabismus quarterly. 2001;16(4):265-70.

10. Chia A, Seenyen L, Long QB. Surgical experiences with two-muscle surgery for the treatment of intermittent exotropia. Journal of AAPOS: the official publication of the American Association for Pediatric Ophthalmology and Strabismus. 2006;10(3):206-11.

11. Zhuang W, Fang S, Fan H, Zhu W, Chen Y, Tang W, et al. Anatomical study of the extraocular check ligament system. Journal of plastic, reconstructive \& aesthetic surgery: JPRAS. 2019;72(12):201726.

12. Clark RA. The Role of Extraocular Muscle Pulleys in Incomitant Non-Paralytic Strabismus. Middle East African journal of ophthalmology. 2015;22(3):279-85.

13. Ke G, Meng Q, Finley T, Wang T, Chen W, Ma W, et al. LightGBM: a highly efficient gradient boosting decision tree. Proceedings of the 31st International Conference on Neural Information Processing Systems; Long Beach, California, USA: Curran Associates Inc.; 2017. p. 3149-57.

14. Véronneau-Troutman S. Prerequisites for strabismus microsurgery. Microscope instruments and sutures. Developments in ophthalmology. 1989;18:24-35.

15. Guyton DL. A small-incision muscle hook for the Parks cul-de- sac approach for strabismus surgery. Binocular vision \& strabismus quarterly. 2005;20(3):147-50.

16. Parks MM. Fornix incision for horizontal rectus muscle surgery. American journal of ophthalmology. 1968;65(6):907-15.

17. Deacon BS, Fray KJ, Grigorian AP, Qureshi HM, Spencer HJ, Lowery RS, et al. Unilateral strabismus surgery in patients with exotropia results in postoperative lateral incomitance. Journal of AAPOS: the official publication of the American Association for Pediatric Ophthalmology and Strabismus. 2014;18(6):572-5.

18. Burian HM, Spivey BE. THE SURGICAL MANAGEMENT OF EXODEVIATIONS. Transactions of the American Ophthalmological Society. 1964;62:276-306.

19. Jeoung JW, Lee MJ, Hwang JM. Bilateral lateral rectus recession versus unilateral recess-resect procedure for exotropia with a dominant eye. American journal of ophthalmology. 2006;141(4):6838. 
20. Donahue SP, Chandler DL, Holmes JM, Arthur BW, Paysse EA, Wallace DK, et al. A Randomized Trial Comparing Bilateral Lateral Rectus Recession versus Unilateral Recess and Resect for Basic-Type Intermittent Exotropia. Ophthalmology. 2019;126(2):305-17.

21. Jin KW, Choi DG. Outcome of two-muscle surgery for large-angle intermittent exotropia in children. The British journal of ophthalmology. 2017;101(4):462-6.

22. Hatt SR, Leske DA, Mohney BG, Brodsky MC, Holmes JM. Fusional convergence in childhood intermittent exotropia. American journal of ophthalmology. 2011;152(2):314-9.

23. Mi J. The surgery age and observe the binocular vision function of Intermittent Exotropia in children. [Master degree]: Xinjiang Medical University; 2014.

24. Romanchuk KG, Dotchin SA, Zurevinsky J. The natural history of surgically untreated intermittent exotropia-looking into the distant future. Journal of AAPOS: the official publication of the American Association for Pediatric Ophthalmology and Strabismus. 2006;10(3):225-31.

25. Adams WE, Leske DA, Hatt SR, Holmes JM. Defining real change in measures of stereoacuity. Ophthalmology. 2009;116(2):281-5.

26. Chew FL, Gesite-de Leon BU, Quah BL. Post-operative strabismus control and motor alignment for basic intermittent exotropia. International journal of ophthalmology. 2016;9(7):1011-5.

27. Armoogum A, Butcher JM. Timely surgery in intermittent and constant exotropia for superior sensory outcome. American journal of ophthalmology. 2001;132(5):803-4.

28. Wu H, Sun J, Xia X, Xu L, Xu X. Binocular status after surgery for constant and intermittent exotropia. American journal of ophthalmology. 2006;142(5):822-6.

29. M.M.Parks. Concomitant exodeviations. Philadelphia: Harper \& Row; 1991 1991. 1-11 p.

30. Burian HM. [Exodeviations. Classification, diagnosis, therapy]. Annali di ottalmologia e clinica oculistica. 1970;96(9):453 - 64.

31. Gunasekeran DV, Ting DSW, Tan GSW, Wong TY. Artificial intelligence for diabetic retinopathy screening, prediction and management. Current opinion in ophthalmology. 2020;31(5):357-65.

32. Jill Hopkins J, Keane PA, Balaskas K. Delivering personalized medicine in retinal care: from artificial intelligence algorithms to clinical application. Current opinion in ophthalmology. 2020;31(5):329-36.

\section{Figures}




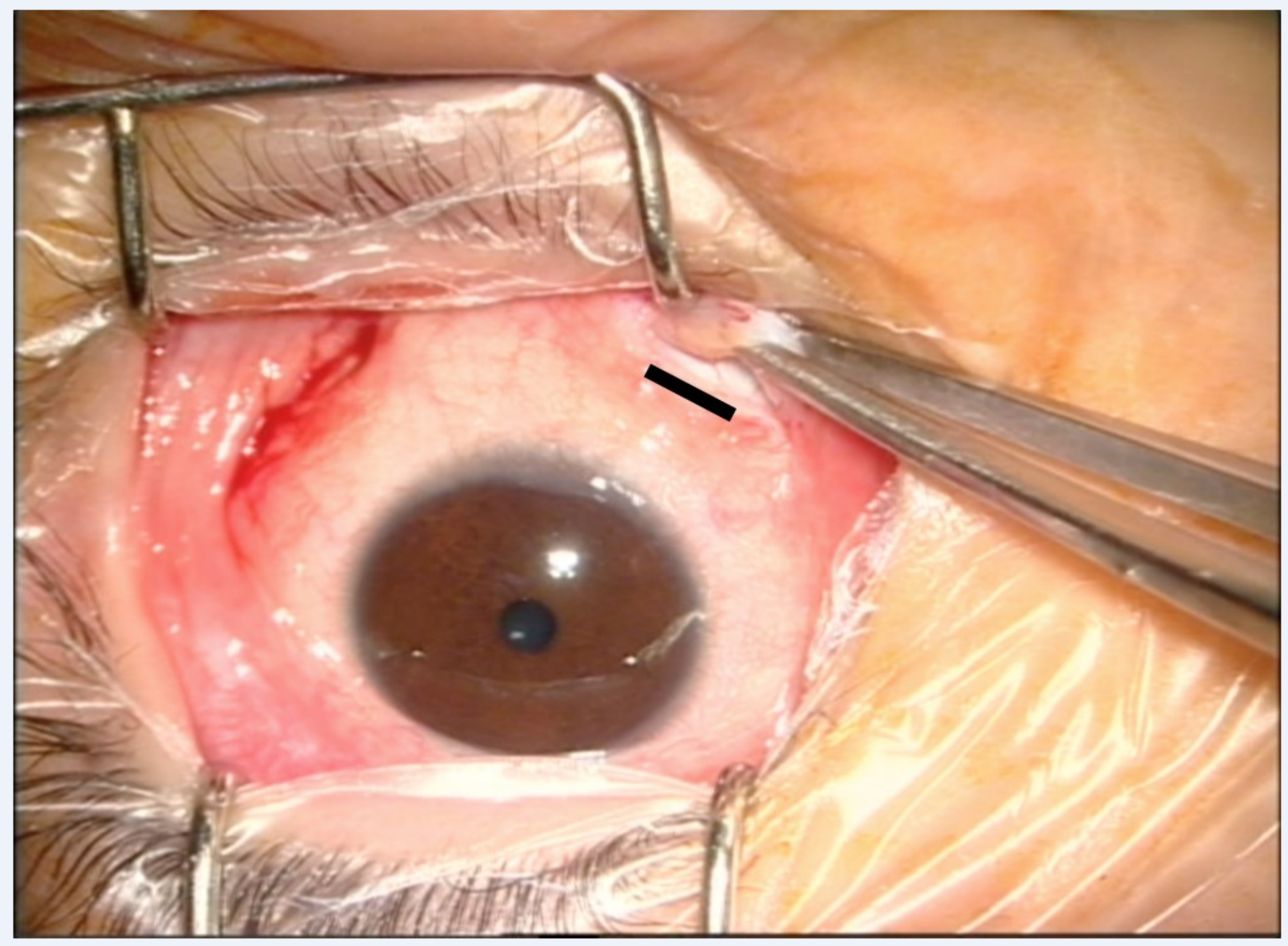

Figure 1

Color photograph of the Parks incision under a surgical microscope. The incision length is approximately $4 \sim 5 \mathrm{~mm}$. 



Figure 2

Color photograph of fully excised check ligaments during the operation under a surgical microscope. (A) shows the superficial check ligament of the external rectus (arrow). (B) shows the check ligament at the lower margin of the external rectus. (C) shows the check ligament at the upper margin of the external rectus. (D) shows complete exposure of the external rectus muscle (arrow). 


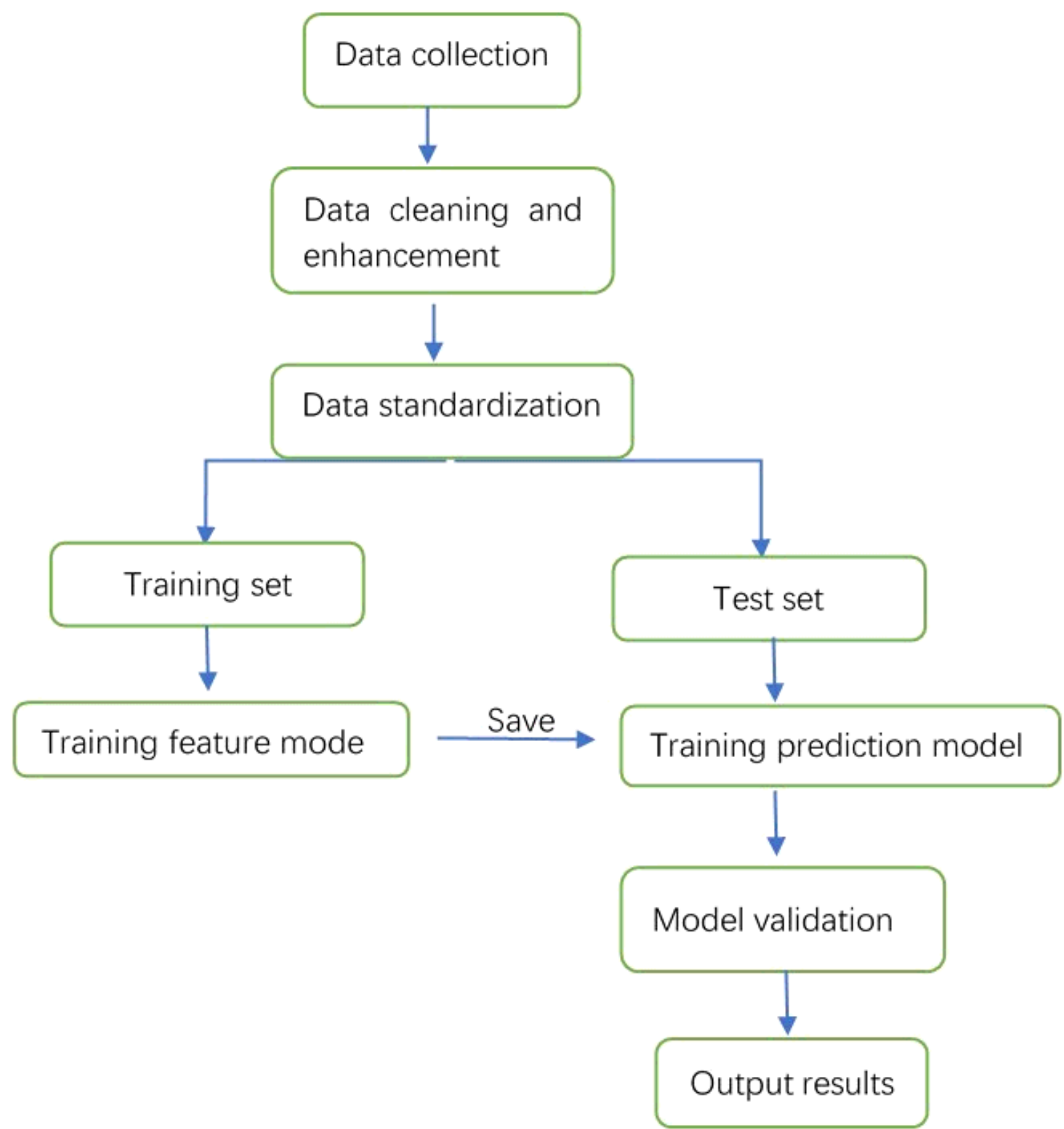

Figure 3

Flow chart of the predictive model. 


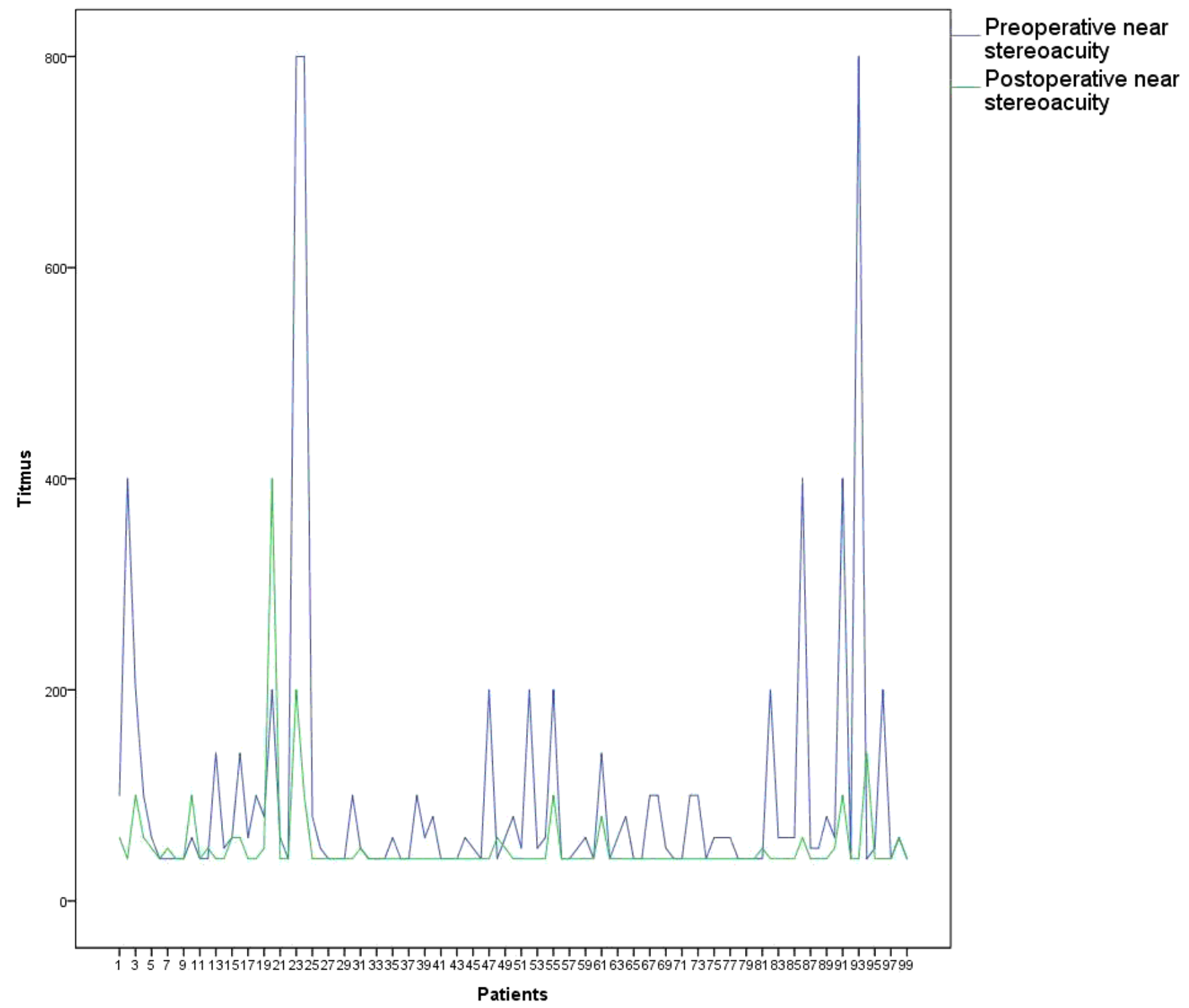

Figure 4

The comparison of near stereoacuity pre- and postoperatively. 
1.2

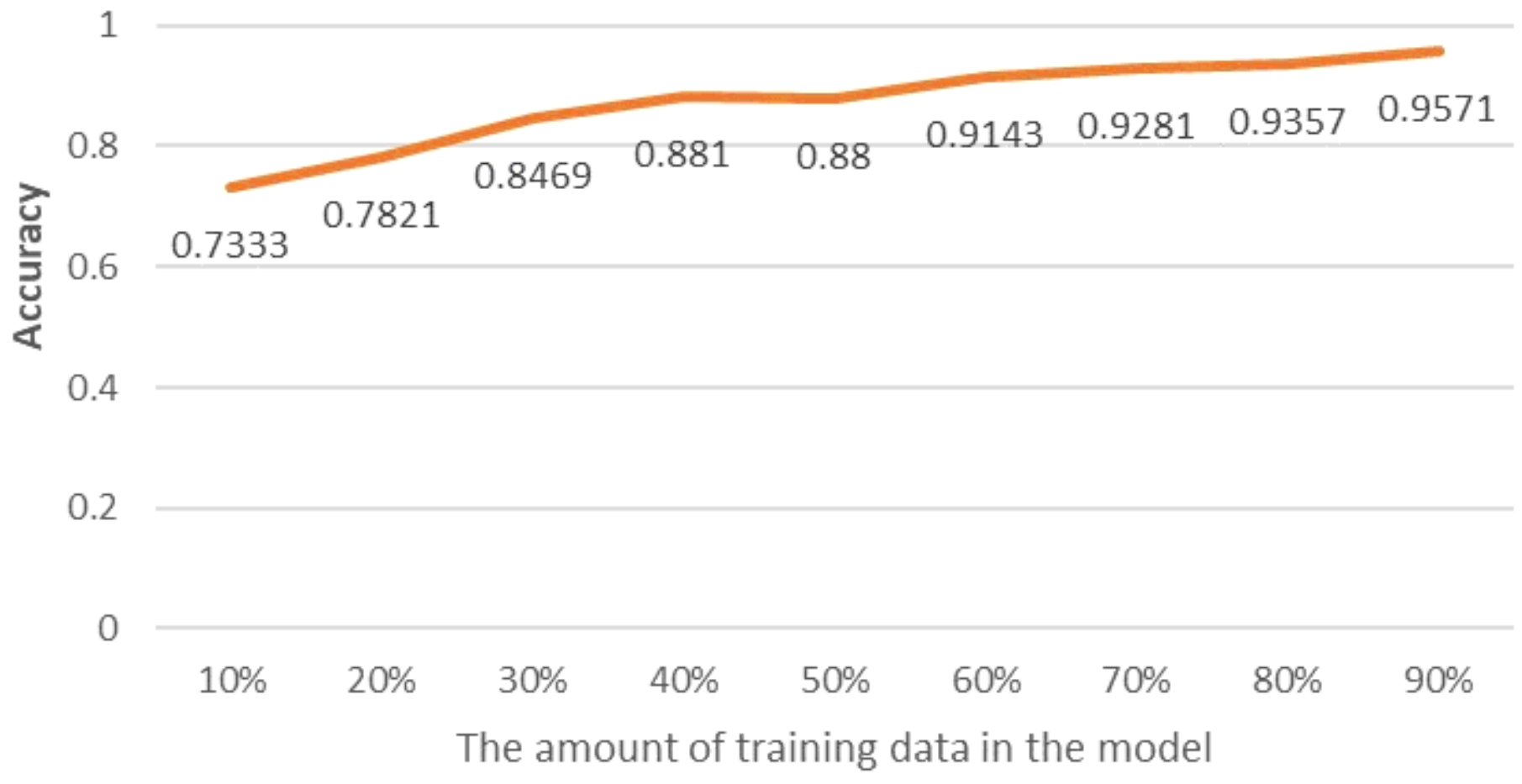

Figure 5

The accuracy of the model changes with the amount of data in training set. 